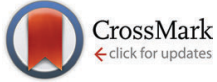

Cite this: Phys. Chem. Chem. Phys., 2016, 18, 24377

Received 17th May 2016 Accepted 8th August 2016

DOI: $10.1039 / c 6 c p 03332 f$

www.rsc.org/pccp

\title{
Micelle formation of a non-ionic surfactant in non-aqueous molecular solvents and protic ionic liquids (PILs) $\dagger$
}

\author{
Emmy C. Wijaya, ${ }^{\text {ab }}$ Frances Separovic, ${ }^{a}$ Calum J. Drummond ${ }^{\mathrm{c}}$ and \\ Tamar L. Greaves*c
}

\begin{abstract}
Many ionic liquids and low molecular weight polar solvents have been reported to support amphiphile self-assembly, with most of these reported for the first time in the last decade. This phenomenon is attributed to the solvophobic effect (analogous to the hydrophobic effect in water). However, to date there has been no systematic study which evaluates micelle formation in a large library of non-aqueous solvents. Here we investigate micelle formation of a non-ionic amphiphile, hexa-ethyleneglycol mono $n$-dodecyl ether, $\mathrm{C}_{12} \mathrm{E}_{6}$, in a diverse range of molecular solvents and protic ionic liquids (PILs). Nine of the 19 non-aqueous molecular solvents investigated, and all four of the PILs, were found to support micelle formation. A link was investigated between the solvent cohesive energy density (as estimated using the Gordon parameter) and both the critical micelle concentration and the related free energy of micellization $\left(\Delta G_{\text {mic }}^{\circ}\right)$. In addition, the chemical structure and liquid mesostructure of the solvent were found to be important factors in the ability of the solvents to support micelle formation.
\end{abstract}

\section{Introduction}

The tendency of surfactants to aggregate in water, widely known as the hydrophobic effect, is significant for diverse applications such as enabling surfactants in soaps and detergent to remove oil-based stains and dirt. At a molecular level, the hydrophobic effect is a key factor in lipid bilayer formation in cell membranes, and maintaining the tertiary structure of proteins. The ability of solvents to promote amphiphile self-assembly is not limited to water, and over the last decades, there have been an increasing number of non-aqueous solvents which have been reported to show the analogous solvophobic effect. Previously we identified 16 commercially available, non-aqueous molecular solvents with solvophobic properties, ${ }^{1}$ increasing to 24 the number of small molecular amines and glycols known to exhibit this property. ${ }^{2-4}$

Ionic liquids (ILs), which are molten salts with melting points below $100^{\circ} \mathrm{C}$, are another non-aqueous solvent group where some have been reported to support amphiphile self-assembly. ${ }^{5-10}$ Some common features shared by many molecular solvents

\footnotetext{
${ }^{a}$ School of Chemistry, Bio21 Institute, University of Melbourne, VIC 3010, Australia

${ }^{b}$ CSIRO Material Science and Engineering, Clayton VIC 3169, Australia

${ }^{c}$ School of Science, College of Science, Engineering and Health, RMIT University, GPO Box 2476, Melbourne VIC 3001, Australia.

E-mail: tamar.greaves@rmit.edu.au

$\dagger$ Electronic supplementary information (ESI) available. See DOI: 10.1039/c6cp03332f
}

and protic ILs (PILs) with a solvophobic effect are the presence of two or more potential hydrogen bonding centres, which enables them to form three-dimensional hydrogen bonded network structures. They usually have few, if any, methylene groups, and often have polar groups such as hydroxyls, both of which increases their solvophobic effect. ${ }^{3}$ While previously few solvents were considered amphiphile self-assembly media, it is now evident that many ILs, including PILs and aprotic ILs, ${ }^{11}$ and molecular solvents share this property. It is anticipated that many more will be identified than are currently known.

One solvent property that is a useful indicator of the solvophobicity is the cohesive energy density. ${ }^{5}$ The cohesive energy density is the energy required to remove a unit volume of molecules completely from their neighbours to infinite separation (an ideal gas state). This is equal to the heat of vaporisation divided by the molar volume. For ILs in which it is not feasible to measure the heat of vaporisation due to negligible vapour pressures, the cohesive energy density can be approximated by taking into account the surface tension at the liquid-vapour interface, $\gamma_{\mathrm{LV}}$, and the molar volume, $V_{\mathrm{m}}$, as shown in eqn (1):

$$
\text { Cohesive energy density }=c \gamma_{\mathrm{LV}} / V_{\mathrm{m}}^{1 / 3}
$$

where $c$ is a scalar quantity. The Gordon parameter, $G$, is obtained from eqn (1) when $c$ is set to 1 , as shown in eqn (2). ${ }^{6,12,13} G$ has been used to give a measure of the cohesive energy density, and 
thus the solvophobic effect for a variety of molecular and ionic liquids. ${ }^{14}$

$$
G=\gamma_{\mathrm{LV}} / V_{\mathrm{m}}^{1 / 3}
$$

Solvents with a high Gordon parameter generally have a higher solvophobic effect and hence are likely to be better at promoting amphiphile self-assembly. ${ }^{1,6,10,14}$ Water has the highest known $G$ value of $2.743 \mathrm{~J} \mathrm{~mol}^{1 / 3} \mathrm{~m}^{-3}$, whereas other non-aqueous solvents have lower $G$ values, with $\mathrm{N}$-tertbutylformamide having the lowest at $0.53 \mathrm{~J} \mathrm{~mol}^{1 / 3} \mathrm{~m}^{-3}$ reported in the literature, while still retaining the ability to promote amphiphile self-assembly. ${ }^{5}$ Although not an absolute measure of the cohesive energy density, $G$ provides a relative comparison of the solvophobic effect between different non-supercritical solvents, and is a simple parameter that can be easily determined for most solvents. The strength of the solvophobic effect varies between solvents and hence the aggregation of amphiphiles within them varies, such as which lyotropic liquid crystal (LLC) mesophases are supported and what is the thermal stability range of the phases. ${ }^{1}$

Studying micelle formation in solvents provides insight into the interactions between amphiphiles and solvents, and a comparison between solvents for their ability to support amphiphile self-assembly. An important micellisation parameter is the critical micelle concentration (CMC), which is defined as the surfactant concentration above which micelles spontaneously form. ${ }^{15}$ Currently, there is no systematic study that evaluates micelle formation in a large library of non-aqueous solvents. Instead the limited CMC data reported for surfactants in non-aqueous solvents is sporadic, and hence minimal comparisons can be made between solvents. However, from the data available it is clear that water is a more favourable media for micelle formation, shown by the smaller CMC values compared to those reported in non-aqueous solvents. ${ }^{2,3,8,16-20}$

In this paper we have investigated the correlation between the solvophobic effect and micelle formation of a non-ionic amphiphile in a broad range of commercially available low molecular weight polar molecular solvents and in four PILs. These solvents were all selected because they have previously been shown to support LLC phases. ${ }^{1}$ A protic ionic liquid, EAN, has been previously shown to support micelle formation. ${ }^{21,22}$ The chemical structure of the amphiphile hexa-ethyleneglycol mono $n$-dodecyl ether $\left(\mathrm{C}_{12} \mathrm{E}_{6}\right)$ is provided in Fig. 1 , and those of the molecular solvents and PILs provided in Fig. 2 and 3, respectively.

\section{Experimental methods}

\section{Materials}

Non-ionic surfactant, hexa-ethyleneglycol mono $n$-dodecyl ether, $\mathrm{C}_{12} \mathrm{E}_{6}$ (Nikkol BL-6SY) was supplied by Nikko Chemicals Co. Ltd,

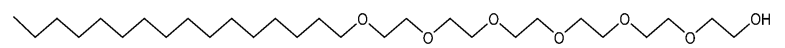

Fig. 1 Structure of the non-ionic surfactant, hexa-ethylene glycol mono $n$-dodecyl ether $\left(\mathrm{C}_{12} \mathrm{E}_{6}\right)$.
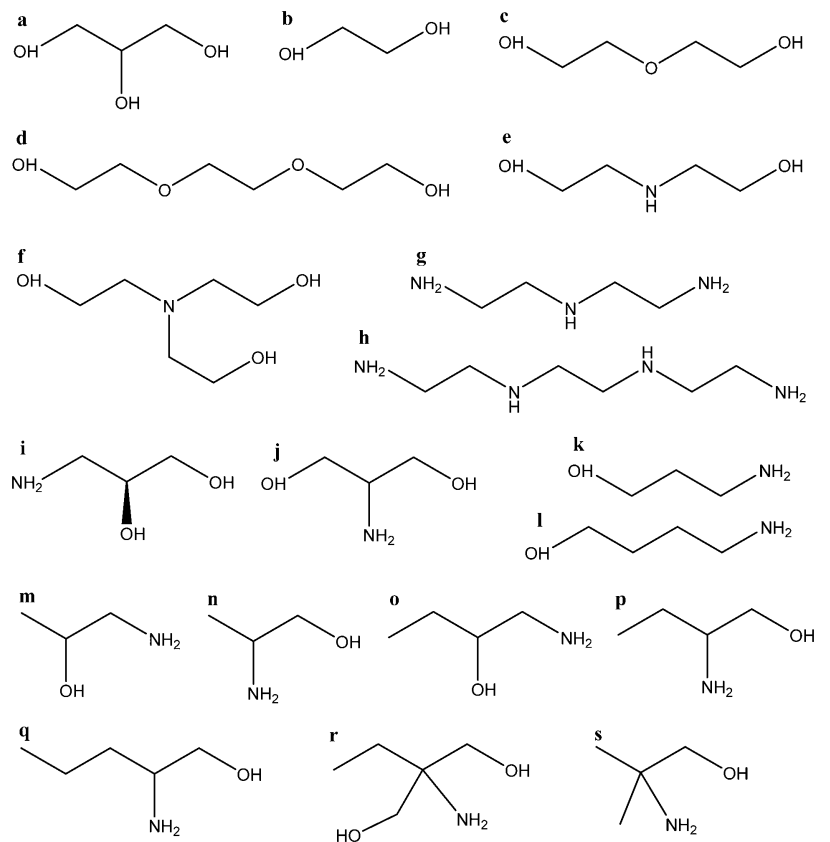

Fig. 2 Chemical structures of the molecular solvents: (a) glycerol, (b) ethylene glycol, (c) diethylene glycol, (d) triethylene glycol, (e) diethanolamine, (f) triethanolamine, (g) diethylene triamine, (h) triethylene tetramine, (i) 3-amino-1,2-propanediol, (j) 2-amino-1,3-propanediol, (k) 3-amino-1propanol, (l) 4-amino-1-butanol, (m) 1-amino-2-propanol, (n) 2-amino-1propanol, (o) 1-amino-2-butanol, (p) 2-amino-1-butanol, (q) 2-amino-1pentanol, (r) 2-amino-2-ethyl-1,3-propanediol, and (s) 2-amino-2-methyl1-propanol.

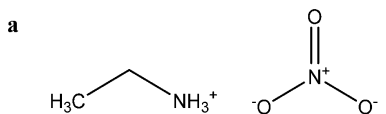

b

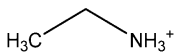<smiles>O=CO</smiles><smiles>O=[N+]([O-])[N-]CCO</smiles>

d<smiles>[NH3+]CCO</smiles><smiles>O=CO</smiles>

Fig. 3 Chemical structures of the PILs: (a) ethylammonium nitrate (EAN), (b) ethylammonium formate (EAF), (c) ethanolammonium nitrate (EOAN), and (d) ethanolammonium formate (EOAF).

Tokyo, Japan, at the highest purity. The 19 molecular solvents were used without further purification: glycerol (Merck, 99\%), ethylene glycol (Sigma Aldrich, 99.8\%), diethylene glycol (Sigma Aldrich, 99\%), triethylene glycol (Sigma Aldrich, 99\%) diethanolamine (AnalaR, 99.5\%), and triethanolamine (Sigma Aldrich, 99.5\%), diethylene triamine (Sigma Aldrich, 99\%), triethylene tetramine (Sigma Aldrich, 97\%), (S)-3-amino-1,2propanediol (Sigma Aldrich, 97\%), 2-amino-1,3-propanediol (Sigma Aldrich, 98\%), 3-amino-1-propanol (Sigma Aldrich, 99+\%), 4-amino-1-butanol (Sigma Aldrich, 98\%), DL-1-amino-2propanol (Sigma Aldrich, 90\%), (R)-(-)-2-amino-1-propanol (Sigma Aldrich, 98\%), 1-amino-2-butanol (Sigma Aldrich, 97\%), 2-amino-1-butanol (Merck, 95\%), DL-2-amino-1-pentanol (Sigma Aldrich, 97\%), 2-amino-2-ethyl-1,3-propanediol (Acros Organics, 97\%), and 2-amino-2-methyl-1-propanol (Fluka, 99\%). 
Ethylamine (Sigma-Aldrich, $70 \mathrm{wt} \%$ ), ethanolamine (Chem Supply, 99.5\%), nitric acid (Chem Supply, 70\% w/w), and formic acid (Merck, 98-100\%) were used without further purifications for synthesis of the PILs. Ethylammonium nitrate (EAN), ethylammonium formate (EAF), ethanolammonium nitrate (EOAN), and ethanolammonium formate (EOAF) were synthesized by dropwise addition of acid to the equimolar amount of amine at a temperature below $0{ }^{\circ} \mathrm{C}$ or above the freezing point of the reaction mixture during synthesis. Excess solvent was removed on a rotary evaporator, and samples were further dried using a benchtop freeze dryer (FreeZone). ${ }^{23}$

The amount of water present in the molecular solvents and the synthesized PILs was measured using a Mettler Toledo DL39 Karl Fischer coulometer.

\section{Experimental}

Solvent-surfactant solutions were prepared using $\mathrm{C}_{12} \mathrm{E}_{6}$ in the various solvents over the concentration range of 0.0001 to $1 \mathrm{M}$. The solutions were mixed with a vortex mixer and left at room temperature for at least one hour before use. All solutions were examined using an optical microscope. Surfactant insolubility was indicated by the presence of "oil-like" droplets. Surface tension measurements were made of all these solutions using a pendant drop KSV Attension Tensiometer. 20 frames of 1 second intervals were acquired for each sample. These were repeated 5 times to ensure consistency. All measurements were performed at room temperature, $22 \pm 1{ }^{\circ} \mathrm{C}$.

Cloud-point measurements were made on solutions of $1 \mathrm{wt} \% \mathrm{C}_{12} \mathrm{E}_{6}$ in each solvent heated to $100{ }^{\circ} \mathrm{C}$, while visual observations were made at what temperature, if any, the solutions went cloudy.

Solutions of $\mathrm{C}_{12} \mathrm{E}_{6}$ in all molecular solvents and PILs for small angle scattering (SAXS) measurements were prepared from two times the CMC up to $10 \mathrm{M}$, with the upper limit depending on the amphiphile solubility in the different solvents. The solutions were then transferred into $1.5 \mathrm{~mm}$ special glass capillaries, and sealed with wax to avoid water absorption and solvent evaporation. Samples were analysed at the SAXS/WAXS beamline at the Australian Synchrotron, Melbourne, Australia. Spectra were acquired over the scattering vector range of $0.01<q<0.6 \AA^{-1}$, with 1 second exposures per sample. The scattering patterns were acquired at $25{ }^{\circ} \mathrm{C}$, which is above the Krafft temperature of $\mathrm{C}_{12} \mathrm{E}_{6}$ of $20{ }^{\circ} \mathrm{C}$ in water. ${ }^{24}$ The $2 \mathrm{D}$ SAXS scattering patterns were converted to $1 \mathrm{D}$ scattering curves with the SAXS I5-id software provided at the Australian Synchrotron. Background scattering curves were obtained from an empty capillary, which was subtracted from the sample scattering patterns. Software SasView ${ }^{25}$ was used to fit the scattering curves in the $q$ range of $0.03-0.3 \AA^{-1}$ with different micelle models.

\section{Micelle scattering models}

The X-ray scattering intensity for micelles is given by eqn (3), where $I(q)$ is the scattering intensity, $K$ is a constant, $N$ is the number of scatterers, $P(q)$ is the form factor, and $S(q)$ the structure factor.

$$
I(q)=K N P(q) S(q)
$$

The form factor $P(q)$ and the structure factor $S(q)$ describe the shape of the micelles and the interactions between micelles, respectively. Interactions between micelles are assumed to be insignificant for low micelle concentrations, i.e., the structure factor is 1 and, thus, the scattered intensity is only described from the form factor. In relatively high concentrations, interactions between micelles needs to be taken into account; thus the structure factor needs to be included. Pedersen provides a comprehensive overview on SAXS scattering modelling that includes a variety of form factors and structure factors for different micelle shapes. ${ }^{26}$

In this work we have used a variety of core-shell micelle models, including spherical, ellipsoidal, rod and disc, to fit the SAXS scattering data for $\mathrm{C}_{12} \mathrm{E}_{6}$ in the different solvents at a variety of concentrations. The models which gave the best fits, and the most logical sequence of parameters on changing concentration, were selected for each solvent- $\mathrm{C}_{12} \mathrm{E}_{6}$ combination. Micelle fitting in this work used a hard sphere structure factor when there was a high concentration of $\mathrm{C}_{12} \mathrm{E}_{6}$ present. This estimates the interparticle structure factor for monodisperse spherical particles which interact through excluded volume, using a Percus-Yevick closure, ${ }^{27}$ and is the most suitable for the non-ionic amphiphile used in this work. In $\mathrm{C}_{12} \mathrm{E}_{6}$, the hydrocarbon core radius must not be greater than the fully extended $C_{12}$ chain length, which is $16.7 \AA{ }^{28}$ according to eqn (4), in which $n_{\mathrm{c}}$ is the number of carbons in the hydrocarbon chain.

$$
l_{\max }=1.5+1.265 n_{\mathrm{c}}
$$

SAXS scattering originates from the electron density contrast between the micelle shell and core, and between the micelle shell and the solvent. The electron scattering length density (SLD) were calculated for the solvents using $\mathrm{SLD}=\rho_{\mathrm{e}} r_{\mathrm{e}}$, where $r_{\mathrm{e}}$ is the electron radius, and $\rho_{\mathrm{e}}=Z / V$, with $Z$ the number of electrons and $V$ the molecular volume. The SLD of the various solvents are provided in Table 3 , and the calculation of the scattering length density for the core and shell of $\mathrm{C}_{12} \mathrm{E}_{6}$ micelles can be found in the ESI. $\dagger$ It was assumed that the hydrocarbon core of micelles is not solvated, thus the SLD of the core was fixed to $7.92 \times 10^{-6} \AA^{-2}$. The solvents were expected to solvate the solvophilic headgroups of $\mathrm{C}_{12} \mathrm{E}_{6}$ and hence the electron density of the micelle shell will vary from those of just the headgroups. Contrast matching occurred to some extent for samples where the SLD of the solvent was comparable to the SLD of the micelle shells. For these samples simple (not core-shell) sphere, ellipsoid and cylinder models were found to fit best.

\section{Results}

\section{CMC and micelle interfacial properties}

The CMC of $\mathrm{C}_{12} \mathrm{E}_{6}$ in water and in nine of the molecular solvents was obtained using pendant drop surface tension measurements, and these are provided in Table 1 along with their interfacial properties. Similarly the values for $\mathrm{C}_{12} \mathrm{E}_{6}$ in the PILs are provided in Table 2 . The initial surface tension values 
Table 1 Gordon parameter, CMC, surface tension of neat solvent $\left(\gamma_{0}\right)$, surface tension at CMC $\left(\gamma_{\mathrm{cmc}}\right)$, surface excess concentration $\left(\Gamma_{\max }\right)$, minimum area per molecule $\left(A_{\mathrm{min}}\right)$, standard free energy of micelle formation $\left(\Delta G_{\text {mic }}^{\circ}\right)$, and standard free energy of adsorption $\left(\Delta G_{\text {ad }}^{\circ}\right)$ for $C_{12} E_{6}$ in molecular solvents at room temperature

\begin{tabular}{|c|c|c|c|c|c|c|c|c|c|}
\hline Solvents & $\begin{array}{l}\text { Water } \\
\text { content } \\
\text { (wt\%) }\end{array}$ & $\begin{array}{l}\text { Gordon } \\
\text { parameter } \\
\left(\mathrm{J} \mathrm{mol}^{1 / 3} \mathrm{~m}^{-3}\right)\end{array}$ & CMC (M) & $\begin{array}{l}\gamma_{0} \\
\left(\mathrm{mN} \mathrm{m}^{-1}\right)\end{array}$ & $\begin{array}{l}\gamma_{\mathrm{cmc}} \\
\left(\mathrm{mN} \mathrm{m}^{-1}\right)\end{array}$ & $\begin{array}{l}\Gamma_{\max }\left(\times 10^{-6}\right. \\
\left.\operatorname{mol~m}^{-2}\right)\end{array}$ & $\begin{array}{l}A_{\min } \\
\left(\AA^{2}\right)\end{array}$ & $\begin{array}{l}\Delta G_{\text {mic }}^{\circ} \\
\left(\mathrm{kJ} \mathrm{mol}^{-1}\right)\end{array}$ & $\begin{array}{l}\Delta G_{\mathrm{ad}}^{\circ} \\
\left(\mathrm{kJ} \mathrm{mol}^{-1}\right)\end{array}$ \\
\hline Water & 100 & $2.743^{38}$ & $7.93 \times 10^{-5}$ & $71.9^{38}$ & 31.89 & 4.34 & 38 & -23.0 & $-1.58 \times 10^{3}$ \\
\hline 3-Amino-1-propanol & 1.70 & 1.07 & $1.56 \times 10^{-1 a}$ & $\begin{array}{l}45.3 \\
44.7^{41}\end{array}$ & 30.80 & 1.66 & 100 & -4.6 & $-1.46 \times 10^{3}$ \\
\hline 4-Amino-1-butanol & 1.88 & 0.97 & $2.45 \times 10^{-1 a}$ & $\begin{array}{l}43.9 \\
43.84^{41}\end{array}$ & 34.20 & 1.88 & 88 & -3.5 & $-0.88 \times 10^{3}$ \\
\hline Diethylene triamine & 0.80 & 0.91 & $2.58 \times 10^{-1 a}$ & $\begin{array}{l}43.4 \\
48.85^{41}\end{array}$ & 33.89 & 2.19 & 76 & -3.4 & $-0.73 \times 10^{3}$ \\
\hline Triethanolamine & 0.14 & 0.91 & $8.07 \times 10^{-2}$ & $\begin{array}{l}46.7 \\
51.48^{41}\end{array}$ & 32.24 & 2.35 & 71 & -6.2 & $-1.05 \times 10^{3}$ \\
\hline $\begin{array}{l}(R)-(-)-2-\text { Amino-1- } \\
\text { propanol }\end{array}$ & 0.15 & 0.90 & $1.87 \times 10^{-1 a}$ & $\begin{array}{l}38.3 \\
47.03^{41}\end{array}$ & 33.58 & 1.28 & 130 & -4.2 & $-0.6 \times 10^{3}$ \\
\hline
\end{tabular}

${ }^{a}$ The Gibbs equation is derived on the basis of an assumption of a low amphiphile concentration. This assumption may no longer be valid when the CMC is greater than $0.1 \mathrm{M}$. However, the values for $\Gamma_{\max }, A_{\min }, \Delta G_{\mathrm{mic}}^{\circ}$ and $\Delta G_{\text {ad }}^{\circ}$ have been included for all samples to enable relative comparisons between them.

of all these solvents, their Gordon parameters $(G)$, and water contents are also provided in Tables 1 and 2. The values obtained in this experiment for the $\mathrm{CMC}$ of $\mathrm{C}_{12} \mathrm{E}_{6}$ in water and in EAN are $1.27 \times 10^{-4} \mathrm{M}$ and $4.46 \times 10^{-2} \mathrm{M}$, which are in good agreement to what has been reported in the literature. ${ }^{8,29}$ The authors are not aware of any CMC measurement of this surfactant in any of the other solvents used in this work.

A representative plot of surface tension with respect to increasing $\mathrm{C}_{12} \mathrm{E}_{6}$ concentration in ethylene glycol is provided in Fig. 4, and the plots for all the other solvents are provided in Fig. S1-S14 of the ESI. $\dagger$ The CMC corresponds to the intersection of the two linear lines. Above this concentration, the surface tension value will no longer undergo significant change.
The pendant drop technique was not feasible for four of the molecular solvents where the surface tension was less than $34 \mathrm{mN} \mathrm{m}^{-1}$, viz. 2-amino-1-butanol $\left(32.2 \mathrm{mN} \mathrm{m}^{-1}\right), 2$-amino-2ethyl-1,3-propanediol $\left(33.35 \mathrm{mN} \mathrm{m} \mathrm{m}^{-1}\right)$, 1-amino-2-butanol (31.1 $\mathrm{mN} \mathrm{m}^{-1}$ ), and 2-amino-1-pentanol $\left(30.4 \mathrm{mN} \mathrm{m}^{-1}\right)$. Furthermore this technique was not applicable for the solvents with high viscosity, i.e., 3-amino-1,2-propanediol (4.3 Pa s) and glycerol (1.41 Pa s), or solids, 2-amino-2-methyl-1-propanol, 2-amino-1,3-propanediol and diethanolamine. An alternative technique of fluorescence, using pyrene to detect the presence of hydrophobic micelle core, ${ }^{30}$ was trialled but due to fluorescence quenching it was not suitable for obtaining the CMC in these remaining solvents, particularly those containing amines. ${ }^{31}$

Table 2 Gordon parameter, CMC, surface tension of neat solvent $\left(\gamma_{0}\right)$, surface tension at CMC $\left(\gamma_{\mathrm{cmc}}\right)$, surface excess concentration $\left(\Gamma_{\text {max }}\right)$, minimum area per molecule $\left(A_{\text {min }}\right)$, standard free energy of micelle formation $\left(\Delta G_{\text {mic }}^{\circ}\right)$, and standard free energy of adsorption $\left(\Delta G_{\text {ad }}^{\circ}\right)$ for $C_{12} E_{6}$ in protic ionic liquids at room temperature

\begin{tabular}{|c|c|c|c|c|c|c|c|c|c|}
\hline Solvents & $\begin{array}{l}\text { Residual water } \\
\text { content (wt } \% \text { ) }\end{array}$ & $\begin{array}{l}\text { Gordon parameter } \\
\left(\mathrm{J} \mathrm{mol}^{1 / 3} \mathrm{~m}^{-3}\right)\end{array}$ & CMC (M) & $\left(\mathrm{mN} \mathrm{m}^{-1}\right)$ & $\begin{array}{l}\gamma_{\mathrm{cmc}} \\
\left(\mathrm{mN} \mathrm{m} \mathrm{m}^{-1}\right)\end{array}$ & $\begin{array}{l}\Gamma_{\max }\left(\times 10^{-6}\right. \\
\left.\operatorname{mol~m}^{-2}\right)\end{array}$ & $A_{\min }\left(\AA^{2}\right)$ & $\begin{array}{l}\Delta G_{\text {mic }}^{\circ} \\
\left(\mathrm{kJ} \mathrm{mol}^{-1}\right)\end{array}$ & $\begin{array}{l}\Delta G_{\mathrm{ad}}^{\circ} \\
\left(\mathrm{kJ} \mathrm{mol}^{-1}\right)\end{array}$ \\
\hline $\begin{array}{l}\text { Ethanolammonium } \\
\text { formate (EOAF) }\end{array}$ & 0.89 & 1.45 & $1 \times 10^{-3 a}$ & & & & & & \\
\hline $\begin{array}{l}\text { Ethylammmonium } \\
\text { nitrate (EAN) }\end{array}$ & 0.70 & $\begin{array}{l}1.060^{10} \\
1.40^{40}\end{array}$ & $4.04 \times 10^{-2}$ & $47.3^{42}$ & 35.7 & 1.41 & 117.65 & -8.0 & -141 \\
\hline $\begin{array}{l}\text { Ethylammmonium } \\
\text { formate (EAF) }\end{array}$ & 0.42 & 0.87 & $1.81 \times 10^{-2}$ & 42 & 30.8 & 3.00 & 55.41 & -9.9 & -594 \\
\hline
\end{tabular}

nitrate (EOAN)

${ }^{a}$ Surface tension measurement was feasible for measuring the changes in surface tension of $\mathrm{C}_{12} \mathrm{E}_{6}$ in EOAF, but due to its high viscosity of $220 \mathrm{nM} \mathrm{m}{ }^{-1}, 42$ surface tension measurements using the pendant drop technique were technically difficult. However, from Fig. S13 in the ESI it can be determined that the CMC value would be around $1 \times 10^{-3} \mathrm{M}$. Since the gradient could not be accurately obtained the interfacial properties could not be determined. 


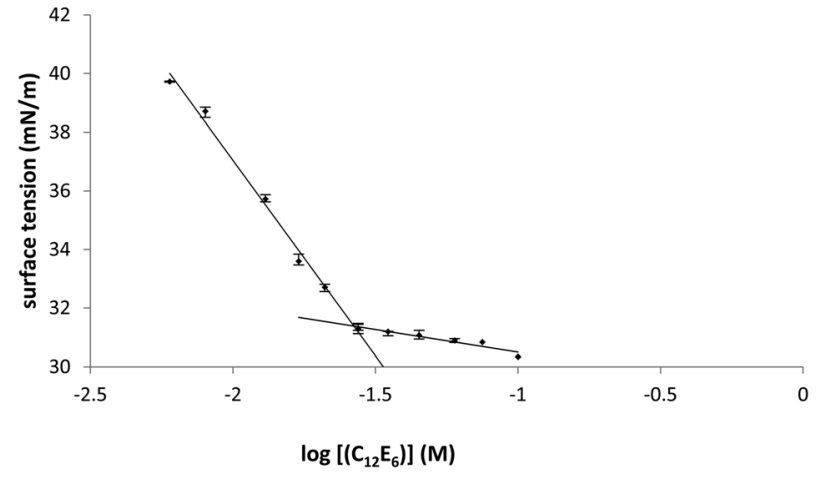

Fig. 4 Determination of $\mathrm{CMC}$ of $\mathrm{C}_{12} \mathrm{E}_{6}$ in ethylene glycol at room temperature using the pendant drop technique.

In addition, visual inspection by optical microscopy showed that $\mathrm{C}_{12} \mathrm{E}_{6}$ in triethylene tetramine formed an insoluble "oil-andwater"-like separation. Consequently the surface tension decrease with increasing concentration, shown in Fig. S10 (ESI $\dagger$ ), was due to reaching a solubility limit rather than a CMC.

\section{Surfactant insolubilities identification with cloud point measurement and optical microscopy}

Cloud point measurements were undertaken in all the molecular solvents and PILs in the presence of $1 \mathrm{wt} \% \mathrm{C}_{12} \mathrm{E}_{6}$. No cloudy appearance was observed in the molecular solvents up to $100{ }^{\circ} \mathrm{C}$, except for water, in which the solution turned cloudy at $46{ }^{\circ} \mathrm{C}$ which was consistent with the literature value of $46.5{ }^{\circ} \mathrm{C}^{32}$ Similarly there was no cloud point below $100{ }^{\circ} \mathrm{C}$ for EAF or EAN, consistent with the literature for EAN. ${ }^{33}$ However, a cloud point at $1 \mathrm{wt} \% \mathrm{C}_{12} \mathrm{E}_{6}$ in EOAN and EOAF was seen immediately at room temperature.

The absence of demixing (cloud point) below $100{ }^{\circ} \mathrm{C}$ for most of the non-aqueous solvents might be related to the solubility of the surfactant in these solvents. A previous study attributed the absence of a cloud point for $\mathrm{C}_{12} \mathrm{E}_{5}, \mathrm{C}_{12} \mathrm{E}_{6}$ and $\mathrm{C}_{12} \mathrm{E}_{8}$ in EAN below $130{ }^{\circ} \mathrm{C}$ to the hydrogen bonded network of EAN enhancing the solubility of the surfactant, and significantly increasing the cloud point temperature. ${ }^{33}$ It is feasible that a similar phenomenon could occur for the non-aqueous solvents used in this investigation. Since they are solvents with polar moieties it is highly likely that there is an interaction with the ethoxy chain, increasing the solubility of surfactant molecules and increasing the cloud point temperature. In contrast, the cloud point seen in $1 \mathrm{wt} \% \mathrm{C}_{12} \mathrm{E}_{6}$ in EOAF and EOAN at room temperature signifies reduced solubility of surfactant in these solvents, which might be due to the hydroxyl end group which could decrease the interaction between the alkyl chain of the surfactant and the solvents, making them more water like, and leading to the presence of a cloud point below $100{ }^{\circ} \mathrm{C}$.

\section{Micelle interfacial properties}

The negative gradient of the surface tension with increasing concentration before the CMC is reached was used to provide insight into the micelle formation and packing of amphiphile molecules at the interface, according to the following equations.
The effectiveness of the surface tension reduction, $\Pi_{\mathrm{cmc}}$, was calculated from eqn (5) for each solvent where the CMC was obtained using the surface tension technique.

$$
\Pi_{\mathrm{cmc}}=\gamma_{0}-\gamma_{\mathrm{cmc}}
$$

where $\gamma_{0}$ is the surface tension of the neat solvent, and $\gamma_{\mathrm{cmc}}$ is the surface tension at the CMC. The minimum molecular area of the $\mathrm{C}_{12} \mathrm{E}_{6}$ surfactant adsorbed at the air-liquid interface, $A_{\text {min }}$, was calculated using a method based on the Gibbs adsorption equation. ${ }^{34-37}$

$$
A_{\min }=10^{20} / N_{\mathrm{A}} \Gamma_{\max }
$$

where $N_{\mathrm{A}}$ is Avogadro's number and $\Gamma_{\max }$ is the maximum surface excess concentration at the air/liquid interface of the non-ionic surfactant, which takes into account the change in surface tension just before the CMC, described by eqn (7).

$$
\Gamma_{\max }=-1 / 2.303 R T\left(\mathrm{~d} \gamma / \mathrm{d} \log \left[\mathrm{C}_{12} \mathrm{E}_{6}\right]\right)
$$

The standard free energy of micellization, $\Delta G_{\text {mic }}^{\circ}$, describes how energetically favourable the micellisation process is, thus giving a measure of the solvophobic interaction.

$$
\Delta G_{\text {mic }}^{\circ}=-R T \ln (\mathrm{CMC})
$$

The standard free energy of adsorption, $\Delta G_{\mathrm{ad}}^{\circ}$, describes the free energy change for the adsorption of amphiphiles to the liquid-air interface, as in eqn (9). These values were calculated for each $\mathrm{C}_{12} \mathrm{E}_{6}$-solvent system, and are shown in Tables 1 and 2 . It should be noted that when $\mathrm{C}_{12} \mathrm{E}_{6}$ had a CMC greater than 0.1 $\mathrm{M}$ assumptions leading to the Gibbs equation may be challenged, but the values are considered to still provide useful indicative information.

$$
\Delta G_{\mathrm{ad}}^{\circ}=\Delta G_{\mathrm{mic}}^{\circ}-\left(\gamma_{0}-\gamma_{\mathrm{cmc}}\right) A_{\mathrm{min}}
$$

The values obtained for $\gamma_{\mathrm{cmc}}, A_{\mathrm{min}}, \Delta G_{\mathrm{mic}}^{\circ}$, and $\Delta G_{\mathrm{ad}}^{\circ}$ of $\mathrm{C}_{12} \mathrm{E}_{6}$ in water and in EAN are in very good agreement with literature values. $^{8}$ In all solvents, $\Delta G_{\text {ad }}^{\circ}$ was calculated to be more negative compared to $\Delta G_{\text {mic }}^{\circ}$, which implies that the adsorption of amphiphiles at the interface is more spontaneous than micelle formation. Thus micelle formation starts after the completion of the adsorption phenomenon at the interface. $\Gamma_{\max }$ was positive in all solvents, which indicates that the concentration of $\mathrm{C}_{12} \mathrm{E}_{6}$ is higher at the liquid-air surface compared to in the bulk. This is typical behaviour of a surface active monomer, with the long hydrophobic chain preferring to be at the liquid-air interface compared to in the bulk solution. ${ }^{43}$ The minimum molecular area, $A_{\min }$, gives insight to the packing density of the surfactant in the interface.

For the molecular solvents, $\mathrm{C}_{12} \mathrm{E}_{6}$ had the smallest $A_{\min }$ of $38 \AA^{2}$ in water, hence the highest packing density, and corresponds to having the highest solvophobic interaction. The non-aqueous molecular solvents all had larger $A_{\min }$ values, and hence higher $\Gamma_{\max }$ values, which can be attributed to a weaker solvophobic interaction, which is directly proportional to $\Delta G_{\text {mic }}^{\circ}$. The larger $A_{\text {min }}$ values in the non-aqueous solvents suggest the surfactant is aligning less perpendicular to the 
liquid-air surface than in water, causing a less tightly packed surface. One possible explanation is that it is due to the reduction of solvent hydrophobicity at the surface, resulting in a drop of surface excess concentration, ${ }^{44}$ which has previously been reported for binary mixtures of water and polar organic solvents. ${ }^{44,45}$

$A_{\text {min }}$ was the largest in 1-amino-2-propanol at $226 \AA^{2}$, which suggests a much weaker solvophobic effect in this solvent, consistent with its low $G$ parameter of $0.86 \mathrm{~J} \mathrm{~mol}^{1 / 3} \mathrm{~m}^{-3}$. The large $A_{\text {min }}$ is attributed to the methyl group in 1-amino-2propanol interacting with the hydrophobic $\mathrm{C}_{12}$ alkyl chain of the surfactant.

The $A_{\min }$ for $\mathrm{C}_{12} \mathrm{E}_{6}$ in triethanolamine and ethylene glycol was $71 \AA^{2}$. Despite its longer chain with an additional alkyl and hydroxyl branch, triethanolamine apparently does not incorporate into the surfactant headgroup. This might be due to its bulkier size leading to steric hindrance minimising its presence at the interface. Also, the terminal hydroxyl group will lead to decreased alkyl solubility, and drive it towards the bulk.

For the PILs, $\Gamma_{\max }$ was exceptionally high in EOAN compared to the other solvents. In fact the calculated $A_{\min }$ of $11 \AA^{2}$ is significantly less than would be the case for a monolayer of molecules packed side by side with no intervening solvent and at $90^{\circ}$ to the interface. Consequently the low $A_{\min }$ value indicates the adsorption of multilayers. $A_{\min }$ was higher in EAN compared to most solvents, except 2-amino-1-propanol. This might be due to the preferential orientation of EAN molecules in which the hydrocarbon moieties face the air. ${ }^{42}$ An interfacial monolayer microenvironment causes surfactants to orient so that the dodecyl chains are perpendicular to the interface and hence the ethylene oxide groups are more parallel to the interface in EAN compared to the other solvents.

\section{Micelle identification by SAXS}

Small and wide-angle X-ray scattering patterns (SAXS/WAXS) were acquired at $25{ }^{\circ} \mathrm{C}$ and $50{ }^{\circ} \mathrm{C}$ for each $\mathrm{C}_{12} \mathrm{E}_{6}$-solvent combination for at least two concentrations above the CMC to confirm if micelles were present and, if so, determine their size and shape. The exceptions were $\mathrm{C}_{12} \mathrm{E}_{6}$ in 2-amino-2-methyl1-propanol, 2-amino-1,3-propanediol, and diethanolamine due to these solvents being solids at room temperature.

A representative series of SAXS patterns is shown in Fig. 5 for $\mathrm{C}_{12} \mathrm{E}_{6}$ in ethylene glycol. The presence of micelles was evident by scattering at $q<0.2 \AA^{-1}$, which increased with increasing surfactant concentration. No scattering due to micelles was observable for $2 \times \mathrm{CMC}$, weak scattering for $5 \times \mathrm{CMC}$, and at $1 \mathrm{M}$ the micelles are sufficiently concentrated for interactions to be observed, identified by the appearance of the characteristic peak at low $q$.

Among the 10 molecular solvents for which a CMC was obtained from pendant drop experiments there were only 6 that showed scattering consistent with micelles. In addition, 2-amino-2-ethyl-1,3-propanediol, one of the four molecular solvents which were not feasible for use with the pendant drop method, showed evidence of micelles. These solvents are listed in Table 3 and the scattering patterns were fitted with a variety

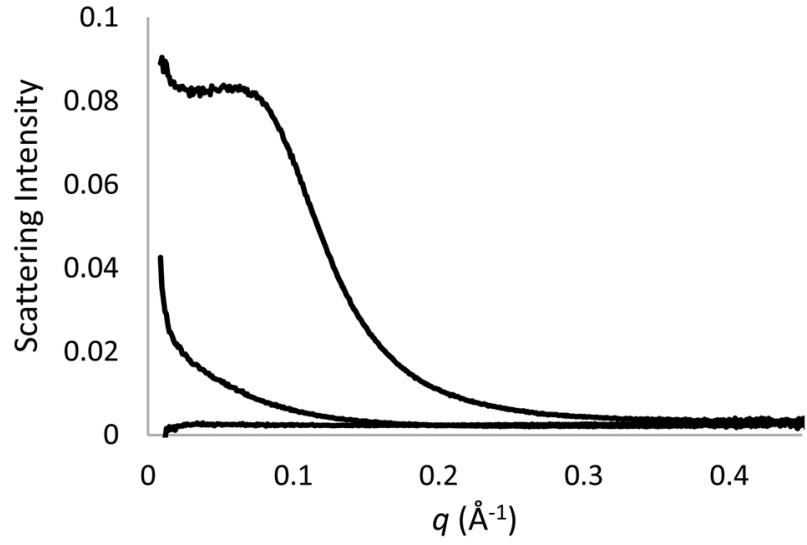

Fig. 5 SAXS patterns of ethylene glycol with $2 \times$ CMC or $0.054 \mathrm{M}$ (bottom), $5 \times \mathrm{CMC}$ or $0.135 \mathrm{M}$ (middle), and $1 \mathrm{M} \mathrm{C}_{12} \mathrm{E}_{6}$ (top).

of micelle models, and the parameters giving the best fits and which were consistent across the different concentrations for each solvent, are provided in Table 3. The SAXS patterns for all $\mathrm{C}_{12} \mathrm{E}_{6}$-solvent solutions are provided in Fig. S15-S21 of the ESI. $f$

The scattering patterns for $\mathrm{C}_{12} \mathrm{E}_{6}$ in all four PILs were consistent with the presence of micelles. However, for EOAF and EOAN, the scattering occurred at concentrations which were similar to the insolubility limit of the $\mathrm{C}_{12} \mathrm{E}_{6}$ in EOAF and EOAN, and consequently these spectra were not fitted. The parameters for the best fits to the micelles in EAN and EAF are provided in Table 3. The SAXS patterns for all $\mathrm{C}_{12} \mathrm{E}_{6}-\mathrm{PIL}$ solutions are provided in Fig. S22 and S23 of the ESI. $\dagger$

The scattering patterns all fitted well to spherical or elliptical micelles, either as simple or core-shell models, as can be seen in Table 3, with the models used for each concentration of $\mathrm{C}_{12} \mathrm{E}_{6}$ clearly specified. Micelles in ethylene glycol and triethanolamine fitted best to simple rather than core-shell models, which was due to similar electron scattering densities between the EO shell and these solvents. The micelles in all the other solvents fitted best to core-shell models. A hardsphere structure factor was included for the higher concentrations of $\mathrm{C}_{12} \mathrm{E}_{6}$ in many of the solvents to account for interactions between micelles. A volume fraction of 0.1 was obtained for those solvents in which hardsphere structure factor was used.

The overall radius of micelles in water, including the core radius and shell thickness, was $33.39 \AA$, which is comparable to the previously reported value of $33 \AA^{28} .^{28}$ Similar work performed by Penfold et al., reported $5 \mathrm{wt} \% \mathrm{C}_{12} \mathrm{E}_{6}$ formed micelles with a radius of $29 \AA^{46},{ }^{46}$ with the smaller radius likely due to the inclusion of a polydispersity factor in their model. Of the nonaqueous molecular solvents, scattering data for micelles have only been reported in ethylene glycol, including small angle neutron scattering (SANS) on $10 \mathrm{wt} \% \mathrm{C}_{12} \mathrm{E}_{8}$ in ethylene glycol, where Seguin et al. reported rod-shaped aggregates with a radius and length of 18 and $127 \AA$, respectively. ${ }^{47}$ These are comparable to our fits for $\mathrm{C}_{12} \mathrm{E}_{6}$ in ethylene glycol of oblate ellipsoids with an equatorial radius of 31-32 $\AA$, and polar radius of 7-8 $\AA$. The differences in radius and length are 
Table 3 SAXS fitting parameters for $\mathrm{C}_{12} \mathrm{E}_{6}$ in molecular solvents and PILs. The form factor for the micelles was kept the same in each solvent

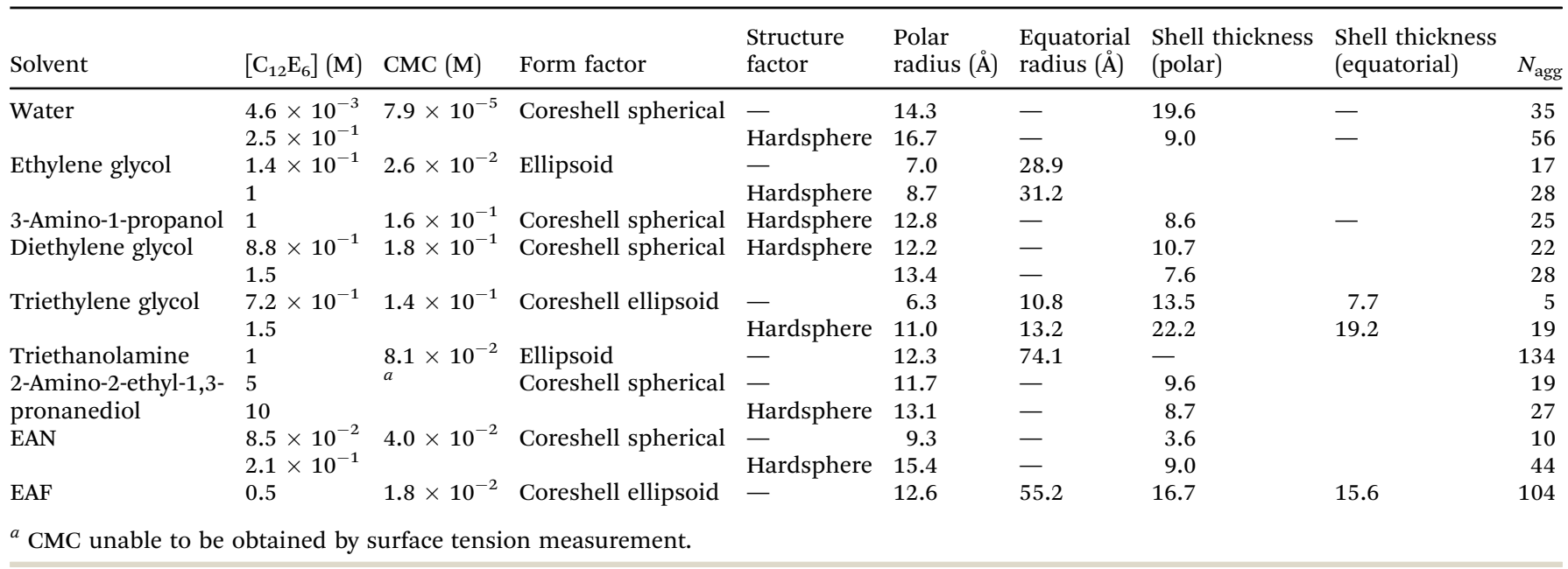

consistent with the larger ethoxy headgroup of their surfactant. In related work, Penfold et al., fitted $5 \% \mathrm{C}_{12} \mathrm{E}_{6}$ in various ethylene glycol-water solutions with a core-shell sphere of radius $16.7 \AA$ and outer shell of $14 \AA^{46} .^{46}$ The use of a core-shell model was in contrast to our fits, and we attribute it to their only including ethylene glycol proportions up to $80 \mathrm{wt} \%$, and hence we propose that for all their compositions there was sufficient water present to have a significant difference between the electron scattering density of the shell and the solvent.

All three EAF concentrations fit well to a coreshell oblate ellipsoid, with no structure factor required. The ellipsoidal micelles were observed to elongate with increasing $\mathrm{C}_{12} \mathrm{E}_{6}$ concentration, becoming more rod-like. The micelles in EAN fitted best to coreshell spheres, with a structure factor present at higher concentrations. Although the electron density between the EO shell and EAN are similar, models that did not include core-shell gave poorer fits. Similar work $^{8}$ performed by Greaves et al. reported ellipsoidal micelles with an effective radius of around $12 \AA$ at $10 \mathrm{wt} \% \mathrm{C}_{12} \mathrm{E}_{6}$, which is comparable to the results in this work. Slight difference in micelle size might be due to the different shape model used. Employing the ellipsoidal model here for the $0.212 \mathrm{M} \mathrm{C}_{12} \mathrm{E}_{6}$ in EAN did not give a good fit.

\section{Discussion}

Here we have investigated the correlation between the solvophobic effect and micelle formation of a non-ionic amphiphile in a broad range of commercially available, low molecular weight polar molecular solvents and easily synthesised PILs. The nonionic amphiphile, $\mathrm{C}_{12} \mathrm{E}_{6}$, was selected because it has a low Krafft temperature $\left(20{ }^{\circ} \mathrm{C}\right)$ in water, ${ }^{24}$ is soluble in a broad range of solvents, and has previously been reported to form micelles in water $^{8}$ and EAN. ${ }^{14,48}$ This investigation enabled comparisons to be made between the non-aqueous solvents, their Gordon parameters and CMCs. We are not aware of any comparable investigation which has used such a broad range of solvents.
The CMC of $\mathrm{C}_{12} \mathrm{E}_{6}$ in all the non-aqueous solvents were at least one order of magnitude higher than in water, with the exception of EOAN, which had a CMC that was about 2.5 times larger than water. This reflects the weaker solvophobic interaction in non-aqueous solutions compared to the hydrophobic effect in aqueous solutions. In general the CMCs in the PILs were lower than in the non-aqueous molecular solvents. It was apparent from the lower Gibb's free energy of micelle formation, $\Delta G_{\text {mic }}^{\circ}$, in non-aqueous solvents compared to water that there was a lower tendency for micellar aggregation. This can be attributed in part to the nonpolar interactions between the alkyl chains of $\mathrm{C}_{12} \mathrm{E}_{6}$ and nonpolar moieties of the solvents. These interactions increase the solubility of $\mathrm{C}_{12} \mathrm{E}_{6}$ and consequently lead to higher CMC values. The rough correlation between the Gibb's free energy of micellisation and the solvophobic effect within the molecular solvents and PILs was shown by plotting $\Delta G_{\text {mic }}^{\circ}$ against the Gordon parameter, $G$ (Fig. 6). It is apparent for the molecular solvents that there is a roughly monotonic relationship, inferring that more energy is generally needed for micelle formation in solvents with lower Gordon parameter values. It is also very clear from Fig. 6 that water has a substantially higher Gordon parameter, and hence higher solvophobic effect, compared to the other solvents used in this work, together with a correspondingly favourable free energy of micellization. The four PILs all had more favourable $\Delta G_{\text {mic }}^{\circ}$ values compared to the molecular solvents with the same Gordon parameters. This suggests that the interaction between cations and anions in PILs are stronger than the interactions in the solvent structures of molecular solvents. Furthermore, the CMC of $\mathrm{C}_{12} \mathrm{E}_{6}$ in EOAF and EOAN of $\sim 1 \times 10^{-3}$ and $2.05 \times 10^{-4} \mathrm{M}$, respectively, was significantly smaller than in EAF and EAN of $4.25 \times 10^{-2}$ and $7.81 \times 10^{-2} \mathrm{M}$, respectively. We attribute this difference to the effect of the hydroxyl group in EOAN and EOAF, which reduced the solubility of $\mathrm{C}_{12} \mathrm{E}_{6}$ in these PILs and hence leads to lower CMC values.

The specific chemical groups present in the molecular solvents and PILs had a large impact on whether or not they 


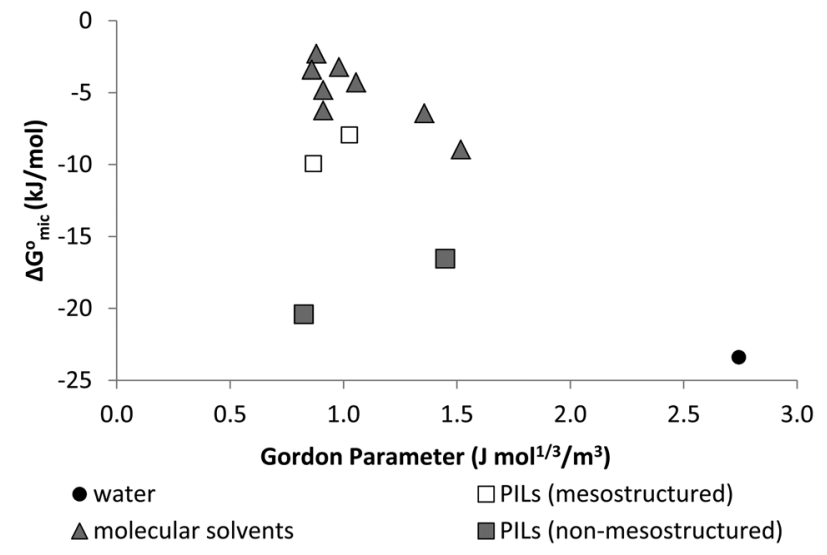

Fig. 6 Gordon parameter versus $\Delta G_{\text {mic }}^{\circ}$ of $\mathrm{C}_{12} \mathrm{E}_{6}$ in the molecular solvents and PILs.

supported the formation of $\mathrm{C}_{12} \mathrm{E}_{6}$ micelles, and if so, on the CMC. Overall, solvents with hydroxyl groups were better solvents for micelle formation compared to those with amine groups, and increasing the proportion of hydrocarbons, particularly methyl or ethyl groups, was highly detrimental. For example, the $\Delta G_{\text {mic }}^{\circ}$ of $\mathrm{C}_{12} \mathrm{E}_{6}$ in hydroxyl containing diethylene glycol and amine containing diethylene triamine was -4.22 and $-3.36 \mathrm{~kJ} \mathrm{~mol}^{-1}$, respectively, showing the higher driving force for micelle formation in the hydroxyl containing solvent, even though they had similar $G\left(0.98 \mathrm{~J} \mathrm{~mol}^{1 / 3} \mathrm{~m}^{-3}\right.$ for the former and $0.91 \mathrm{~J} \mathrm{~mol}^{1 / 3} \mathrm{~m}^{-3}$ for the latter).

Addition of a methylene group increased $\Delta G_{\text {mic }}^{\circ}$ from $-4.60 \mathrm{~kJ} \mathrm{~mol}^{-1}$ in 3-amino-1-propanol to $-3.48 \mathrm{~kJ} \mathrm{~mol}^{-1}$ in 4-amino-1-butanol, due to the increased solubility of the amphiphile. This trend was also seen with $\Delta G_{\text {mic }}^{\circ}$ going from -9.00 to $-4.22 \mathrm{~kJ} \mathrm{~mol}^{-1}$ from ethylene glycol to diethylene glycol with the increased proportion of methylene. The $\Delta G_{\text {mic }}^{\circ}$ of $\mathrm{C}_{12} \mathrm{E}_{6}$ in triethylene glycol did not continue this trend, with its value of $-4.81 \mathrm{~kJ} \mathrm{~mol}^{-1}$ lying between those of ethylene glycol and diethylene glycol. We suggest this may be due to steric hindrance preventing the alkyl chains of the amphiphile to interact with the hydrocarbon portion of the solvent.

The effect of increasing the alkyl chain length on the solvent had a more dramatic effect in reducing the solvent ability to support amphiphile self-assembly compared to increased methylene groups with terminal polar groups. This could most clearly be observed since no molecular solvent which had a terminal ethyl group supported amphiphile self-assembly. For example, the methyl containing 1-amino-2-propanol and 2-amino-1-propanol both supported micelles, whereas their ethyl and propyl counterparts of 1-amino-2-butanol, 2-amino1-butanol and 2-amino-1-pentanol did not. The exception was 2-amino-2-ethyl-1,3-propanediol where scattering from micelles was detected using SAXS. The solvents 1-amino-2-butanol, 2-amino-1-butanol and 2-amino-1-pentanol have previously been shown to form a mesostructure consisting of polar and non-polar domains, whereas 2-amino-2-ethyl-1,3-propanediol did not. ${ }^{1}$ Consequently it is likely that the non-polar domains within the mesostructured solvents may enhance the solubility of amphiphiles and decrease the likelihood of micelle formation. In 2-amino-2-ethyl-1,3-propanediol the two hydroxyl and one amine group are likely to form a multiple hydrogen bonded network with steric hindrance making the segregation of the alkyl chains energetically unfavourable. Interestingly, contrary to the trend found in molecular solvents, EAN and EAF, which contain ethyl groups, were found to support micelle formation. This further shows the exceptional properties that PILs have compared to the molecular solvents.

The driving force for micelle formation of $\mathrm{C}_{12} \mathrm{E}_{6}$ in molecular solvents was significantly weaker than in the PILs. This was shown by the higher $\Delta G_{\text {mic }}^{\circ}$ in molecular solvents. Since the nonionic surfactant $\mathrm{C}_{12} \mathrm{E}_{6}$ will not be affected by the ionic behavior of the PILs, we suggest this is due to that stronger solvent-solvent interactions in PILs compared to in molecular solvents. Interestingly, the magnitude of $A_{\min }$ in these molecular solvents was found to be within the same range as the $A_{\min }$ in EAN and EAF (between 50 to $120 \AA^{2}$ ), indicating that these molecular solvents and two PILs also behave as co-surfactants, and are incorporated to some extend within the micelle.

The effect of solvent mesostructure was also clearly observable for the four PILs. The hydroxyl containing EOAF and EOAN are not mesostructured, whereas EAN and EAF are ref. 49 and 50. The formation of non-polar domains would result in some solvation of the hydrocarbon of the amphiphiles and decrease the driving force for micelles to form, which is evident from the higher $\Delta G_{\text {mic }}^{\circ}$ in EAF and EAN compared to EOAN and EOAF. ${ }^{51}$ In addition, the larger $A_{\min }$ in EAF and EAN suggests that the alkyl chains of the cation behave to some extent as co-surfactants. In contrast, the non-structured EOAN, has a significantly higher $\Gamma_{\max }$ and lower $A_{\min }$ compared to EAF, EAN, water and the other molecular solvents. The particularly low $A_{\text {min }}$ value of $11 \AA^{2}$ indicates that the $\mathrm{C}_{12} \mathrm{E}_{6}$ formed multilayers at the air-liquid interface prior to micellization. It is presumed that similar amphiphile behavior would be present in EOAF.

The presence of micelles detected by SAXS can be related to solvent solvophobicity. In most cases, micelle features were only observed at concentration $>0.1 \mathrm{M}$ for the non-aqueous solvents, which is higher than a concentration equivalent to $5 \times$ CMC. The clearest example was seen in 2-amino-2-ethyl-1,3-pronanediol in which micelles were only observed at a $\mathrm{C}_{12} \mathrm{E}_{6}$ concentration of $5 \mathrm{M}$, and the characteristic peak due to inter-micellar interactions was observed at $10 \mathrm{M}$. The shell thickness gives insight into the interaction between the EO shell and solvents, and was found to be the highest in water compared to either molecular solvents or PILs. This might be due to water being a better solvent for the EO headgroups, allowing them to extend more freely. It may also be an artefact due to the significantly higher contrast between the electron scattering length density of the solvent and the shell. Alternatively, the EO chains could be more compact in the other solvents, be it in the molecular solvents or PILs. This would indicate less interaction between the EO shell with the solvents, as found by Atkin $e t$ al., in EAN. ${ }^{52}$

The aggregation number $\left(N_{\text {agg }}\right)$ describes the number of surfactant molecules in micelles once the CMC has been 
reached. In general $N_{\text {agg }}$ was observed to increase as the surfactant concentration increased (Table 3). Exceptions were micelle sizes in ethylene glycol and 2-amino-2-ethyl-1,3pronanediol in which micelle dimensions, and consequently $N_{\text {agg }}$, underwent a slight reduction with increasing surfactant concentration. This shows that in some cases micelle size does not depend on surfactant concentration. We consider that the micelles may have reached their maximum size due to the high surfactant concentrations used in this study. Consequently the effect of solvent solvophobicity and solvent chemical constituents on micelle size was difficult to determine from the limited data obtained and the variety of CMC values in these solvents. The solvents that can be compared are ethylene glycol, diethylene glycol and triethylene glycol in which the data of micelle size and $N_{\text {agg }}$ are available at $5 \times$ CMC. For these three solvents, $N_{\text {agg }}$ decreased in the order EG $>$ DG $>$ TG, which corresponds to a decrease in solvent solvophobicity. In the case of the PILs, data for micelles at $2 \times$ and $5 \times$ CMC in EAN and EAF were obtained. From Table 3, micelles in EAF, which had lower $G$ (and thus lower solvent solvophobicity) than EAN, had smaller micelle size and lower $N_{\text {agg }}$ value. This also suggests that PILs with higher solvophobicities result in larger micelle sizes and $N_{\text {agg }}$ values.

Overall, as indicated by lower CMC and $\Delta G_{\text {mic }}^{\circ}$ values, PILs used in this investigation were better at supporting micelle formation than the non-aqueous molecular solvents. Moreover, micelles were detected in EAN and EAF at a surfactant concentration as low as $2 \times \mathrm{CMC}$, in which the size was comparable to micelles formed in $1 \mathrm{wt} \%$ in water. Interestingly, EAN, EAF, EOAF and EOAN had Gordon parameter values in the same range as for the molecular solvents. The ionic interaction between positive and negative ions in PILs are likely to be much stronger compared to interactions between molecular solvent, which would translate to lower CMC values, $\Delta G_{\text {mic }}^{\circ}$ and smaller micelle sizes.

\section{Conclusions}

The solvophobic effect and its effect on amphiphile selfassembly were explored by studying the ability of several nonaqueous polar organic solvents to support micelle formation. Generally, an increase in solvophobicity (indicated by higher $G$ ) resulted in lower CMC, and consequently lower $\Delta G_{\text {mic }}^{\circ}$ values. Calculation of interfacial properties suggest that, compared to water, the selected non-aqueous solvents cause surfactant molecules to align less perpendicular to the air-water interface, as indicated by larger $A_{\text {min }}$, or may form multilayers, as indicated by significantly smaller $A_{\text {min }}$ values. Micelle size and $N_{\text {agg }}$ were found to decrease as $G$ decreased. This shows that solvent solvophobicity is a significant contributor to micelle formation and that $G$ is a useful parameter for predicting the probability of amphiphile self-assembly in a solvent. However, there are other factors that need to be considered, such as solvent mesostructure and specific chemical moieties. This study also shows that, based on lower CMCs, select PILs are better solvents for promoting amphiphile self-assembly compared to the molecular solvents. This indicates that the ionic interaction between cations and anions in PILs are stronger when compared to the specific hydrogen-bond interactions between molecular solvents.

\section{Acknowledgements}

ECW is the recipient of a University of Melbourne International Fee Remission Award, a CSIRO Materials Science and Engineering PhD Studentship and Albert Shimmins Writing Up Award. This research was undertaken in part on the SAXS/WAXS beamline at the Australian Synchrotron, Melbourne, Australia.

\section{Notes and references}

1 E. C. Wijaya, T. L. Greaves and C. J. Drummond, Faraday Discuss., 2013, 167, 191.

2 A. Ray, J. Am. Chem. Soc., 1969, 91, 6511.

3 A. Ray, Nature, 1971, 231, 313.

4 T. L. Greaves, A. Weerawardena and C. J. Drummond, Phys. Chem. Chem. Phys., 2011, 13, 9180.

5 T. L. Greaves and C. J. Drummond, Chem. Soc. Rev., 2013, 42, 1096.

6 T. L. Greaves, A. Weerawardena, C. Fong and C. J. Drummond, J. Phys. Chem. B, 2007, 4082.

7 T. L. Greaves and C. J. Drummond, Chem. Soc. Rev., 2008, 37, 1709.

8 T. L. Greaves, S. T. Mudie and C. J. Drummond, Phys. Chem. Chem. Phys., 2011, 13, 20441.

9 T. L. Greaves, A. Weerawardena, I. Krodkiewska and C. J. Drummond, J. Phys. Chem. B, 2008, 112, 896.

10 T. L. Greaves, A. Weerawardena, C. Fong and C. J. Drummond, Langmuir, 2007, 23, 402.

11 I. A. Sedov and B. N. Solomonov, Fluid Phase Equilib., 2016, 425, 9.

12 D. Kabir ud and P. A. Koya, Langmuir, 2010, 26, 7905.

13 P. Ajmal Koya, D. Kabir ud and K. Ismail, J. Solution Chem., 2012, 41, 1271.

14 D. F. Evans, Langmuir, 1988, 4, 3.

15 D. Yu, F. Huang and H. Xu, Anal. Methods, 2012, 4, 47.

16 H. N. Singh, S. M. Saleem, R. P. Singh and K. S. Birdi, J. Phys. Chem., 1980, 84, 2191.

17 M. S. Akhter and S. M. Alawi, Colloids Surf., A, 2003, 219, 281.

18 A. Lattes and I. Rico, Colloids Surf., 1989, 35, 221.

19 M. S. Akhter, Colloids Surf., A, 1999, 150, 25.

20 M. S. Akhter, S. M. Alawi and A. N. Bose, Colloids Surf., A, 1995, 173.

21 D. F. Evans, A. Yamauchi, R. Roman and E. Z. Casassa, J. Colloid Interface Sci., 1982, 88, 89.

22 D. F. Evans, A. Yamauchi, G. J. Wei and V. A. Bloomfield, J. Phys. Chem., 1983, 87, 3537.

23 T. L. Greaves, A. Weerawardena, I. Krodkiewska and C. J. Drummond, J. Phys. Chem. B, 2008, 112, 896.

24 T. Inoue, Y. Higuchi and T. Misono, J. Colloid Interface Sci., 2009, 338, 308. 
25 SAS Institute; SAS: The Power to Know 2016, http:/www.sas. com/en_au/home.html, date accessed 5 May 2016.

26 J. S. Pedersen, Adv. Colloid Interface Sci., 1997, 70, 171.

27 J. K. Percus and G. J. Yevick, Phys. Rev., 1958, 110, 1.

28 C. Tanford, The Hydrophobic Effect: Formation of Micelles and Biological Membranes, Wiley, New York, 1973.

29 R. Dong and J. Hao, Chem. Rev., 2010, 110, 4978.

30 J. Aguiar, P. Carpena, J. A. Molina-Bolívar and C. Carnero Ruiz, J. Colloid Interface Sci., 2003, 258, 116.

31 B. K. Paul and S. P. Moulik, Ionic liquid-based surfactant science: formulation, characterization and applications, John Wiley \& Sons, Hoboken, New Jersey, 2015.

32 K. S. Sharma, S. R. Patil and A. K. Rakshit, Colloids Surf., A, 2003, 219, 67.

33 M. U. Araos and G. G. Warr, J. Phys. Chem. B, 2005, 109, 14275.

34 C. J. Drummond, G. G. Warr, F. Grieser, B. W. Ninham and D. F. Evans, J. Phys. Chem., 1985, 89, 2103.

35 E. Matijevic and B. A. Pethica, Trans. Faraday Soc., 1958, 54, 1382.

36 M. J. Rosen and S. B. Sulthana, J. Colloid Interface Sci., 2001, 239, 528.

37 C. Fong, T. L. Greaves, T. W. Healy and C. J. Drummond, J. Colloid Interface Sci., 2015, 449, 364.

38 D. J. Mitchell, G. J. T. Tiddy, L. Waring, T. Bostock and M. P. McDonald, J. Chem. Soc., Faraday Trans. 1, 1983, 79, 975 .
39 T. Wolff and G. von Bünau, Ber. Bunsen-Ges. Phys. Chem., 1984, 88, 1098.

40 A. H. Beesley, D. F. Evans and R. G. Laughlin, J. Phys. Chem., 1988, 92, 791.

41 C. L. Yaws, Thermophysical Properties of Chemicals and Hydrocarbons, William Andrew Inc., Norwich, N.Y., 2008.

42 T. L. Greaves, A. Weerawardena, C. Fong, I. Krodkiewska and C. J. Drummond, J. Phys. Chem. B, 2006, 110, 22479.

43 I. Mukherjee, S. P. Moulik and A. K. Rakshit, J. Colloid Interface Sci., 2013, 394, 329.

44 S. Das, S. Mondal and S. Ghosh, J. Chem. Eng. Data, 2013, 58, 2586-2595.

45 S. Sinha, D. Tikariha, J. Lakra, A. Tiwari, S. Saha and K. Ghosh, J. Surfactants Deterg., 2015, 18, 629.

46 J. Penfold, E. Staples, I. Tucker and P. Cummins, J. Colloid Interface Sci., 1997, 185, 424.

47 C. Seguin, J. Eastoe, R. Clapperton, R. K. Heenan and I. Grillo, Colloids Surf., A, 2006, 282, 134.

48 M. U. Araos and G. G. Warr, Langmuir, 2008, 24, 9354.

49 R. Atkin and G. G. Warr, J. Phys. Chem. B, 2008, 112, 4164.

50 T. L. Greaves, D. F. Kennedy, N. Kirby and C. J. Drummond, Phys. Chem. Chem. Phys., 2011, 13, 13501.

51 T. L. Greaves, D. F. Kennedy, S. T. Mudie and C. J. Drummond, J. Phys. Chem. B, 2010, 114, 10022.

52 O. Werzer, G. G. Warr and R. Atkin, Langmuir, 2011, $27,3541$. 